\title{
Unsupervised Learning Method for SAR Image Classification Based on Spiking Neural Network
}

\author{
Jiankun Chen ${ }^{\mathrm{a}, \mathrm{c}, \mathrm{d}}$, Xiaolan Qiu ${ }^{\mathrm{a}, \mathrm{d}}$, Chuanzhao Han ${ }^{\mathrm{b}}$, Yirong $\mathrm{Wu}^{\mathrm{d}}$ \\ a The Key Laboratory of Technology in Geo-spatial Information Processing and Application System, Chinese Academy \\ of Sciences, Beijing 100190, China \\ ${ }^{\mathrm{b}}$ Beijing Institute of Remote Sensing Information, Beijing 100192, China \\ ${ }^{c}$ The University of Chinese Academy of Sciences, Beijing 100049, China \\ d Aerospace Information Research Institute, Chinese Academy of Sciences, Beijing 100190, China
}

\begin{abstract}
Recent neuroscience research results show that the nerve information in the brain is not only encoded by the spatial information. Spiking neural network based on pulse frequency coding plays a very important role in dealing with the problem of brain signal, especially complicated space-time information. In this paper, an unsupervised learning algorithm for bilayer feedforward spiking neural networks based on spike-timing dependent plasticity (STDP) competitiveness is proposed and applied to SAR image classification on MSTAR for the first time. The SNN learns autonomously from the input value without any labeled signal and the overall classification accuracy of SAR targets reached $84.2 \%$. The experimental results show that the algorithm adopts the synaptic neurons and network structure with stronger biological rationality, and has the ability to classify targets on SAR image. Meanwhile, the feature map extraction ability of neurons is visualized by the generative property of SNN, which is a beneficial attempt to apply the brain-like neural network into SAR image interpretation.
\end{abstract}

\section{Introduction}

Artificial neural networks (ANN) is a mathematical model to simulate the structure and function of the biological neural system. In recent years, the research work of ANN has been in-depth and has made great progress in the fields of pattern recognition, intelligent robots, automatic control, etc. The current ANN, such as convolutional neural networks and fully-connected feedforward neural networks, receive and output continuous values in the learning process. Although modern neural networks have enabled us to make breakthroughs in many fields, they are biologically imprecise and the simulation of the biological brain neurons is limited.

Spiking neural network (SNN) is so-called the third generation of artificial neural network and it has been theorized to be a more powerful and biologically realistic network model. Compared with the first and second generation of artificial neural models, the data processing of SNN adopts the pulse sequence including time, frequency, space, and other information, which greatly improves the computing ability and can process complex spatiotemporal information. So, it can better simulate different kinds of neuron signals, make it closer to the biological neural system, and greatly improve biological authenticity.

The spiking neural network has attracted extensive attention as soon as it appeared. Many renowned research teams have carried out long-term and detailed research on it. Professor Diesmann [1] of JULICH research center in Germany and other professors have explored the discharge activities of the SNN, provided a framework for classifying and evaluating different models of plasticity and developed the simulation platform NEST. Switzer expert Diehl [2] analyzed the effects of converting deep ANNs into SNNs with respect to the choice of parameters for spiking neurons such as firing rates and thresholds. Yi Zeng [3] focused on biological plausible methodologies and proposed Voltage-driven Plasticity-centric SNN (VPSNN) which includes four steps, namely: feed forward inference, unsupervised equilibrium state learning, supervised last layer learning and passively updating synaptic weights based on spike-timing dependent plasticity (STDP). He built a three-layer impulsive neural network and received the classification accuracy of $98.52 \%$ on MNIST. With the continuous improvement of biological neural model theory and the absorption of the ANN method, the potential and advantages of SNNs can be further extended and maybe exceed the other types of ANN.

In this paper, we propose a spiking neural network for SAR target classification. By using an unsupervised learning method based on STDP, the neural network extracts the features of the input image and gives out a prediction of category. That is, SNN learns autonomously from the input value without any labeled signal and modulates the synaptic connection strength between presynaptic and postsynaptic neurons by STDP rule according to the order of action potential. This method is developed from Hebbian learning mechanism with high biological rationality and biological characteristics, purposing to reduce the error between standard output and actual output in order to get more accurate results. This is the first attempt to apply SNN to SAR image classification to our knowledge.

\section{Architecture of SNN}

The network structure proposed in this paper is a bilayer 


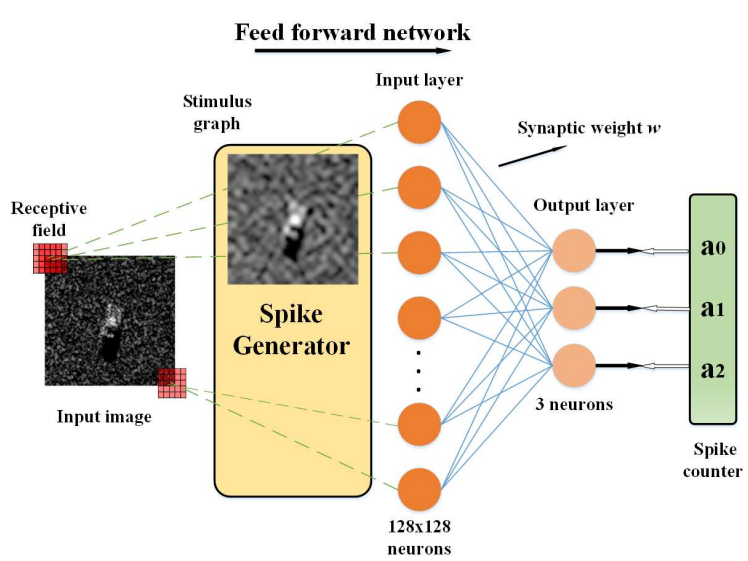

Figure 1 The architecture of bilayer feedforward SNN.

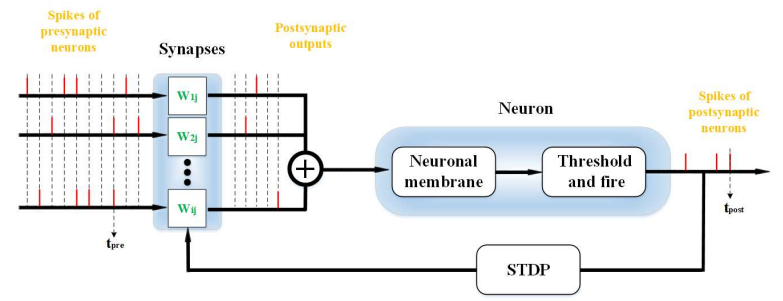

Figure 2 Principle of the change of neuron membrane potential and the passive update of synaptic weights.

spiking neural network (SNN) with the function of three categories classification of images. The first layer is the input layer which contains $128 \times 128$ neurons, and the second layer is the output layer with only three neurons. In the experiment, the input image is first convoluted by a $5 \times$ 5 receptive field as the stimulus graph. Then spike generator transmits a timing pulse pixel by pixel according to the pixel value of stimulus graph and directly maps it to the first layer. The input information of the timing pulse sequence is transmitted to the second layer through the initialed synaptic weights. In each neuron, the change of membrane potential and the generation of action potential conform to biological rules. With the continuous learning of SNN, all synaptic weights are passively updated according to STDP rule. A counter of spike number is connected with the end of the output layer, which records the total number of spikes generated by each output neuron in the timing period corresponding to the pixel of input image. The neuron with the most spike times wins and is marked as a category of the current image. The architecture of SNN and the learning principle of neurons used in this paper are shown in the figure 1 and figure 2.

\section{$3 \quad$ Learning mechanism of Spiking Neural Network based on STDP}

\subsection{Synaptic structure and dynamic pulse neuron}

Leaky-Integrated and fire (LIF) model is the most commonly used as the neuron model of SNN which can well capture the firing characteristics of biological neuron. In the absence of a stimulus, the membrane possesses a resting potential. Every input spike from connected
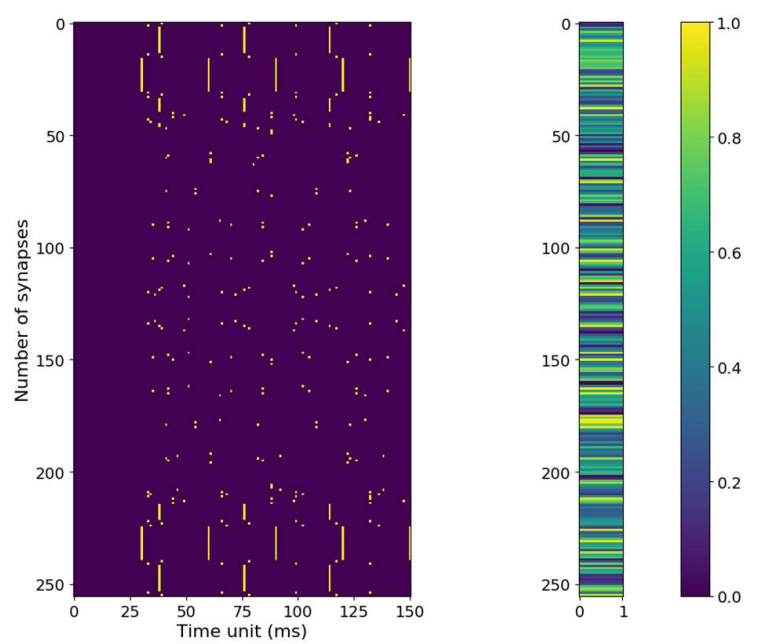

Figure 3 A set of input pulses (left) and connecting synaptic weights (right).

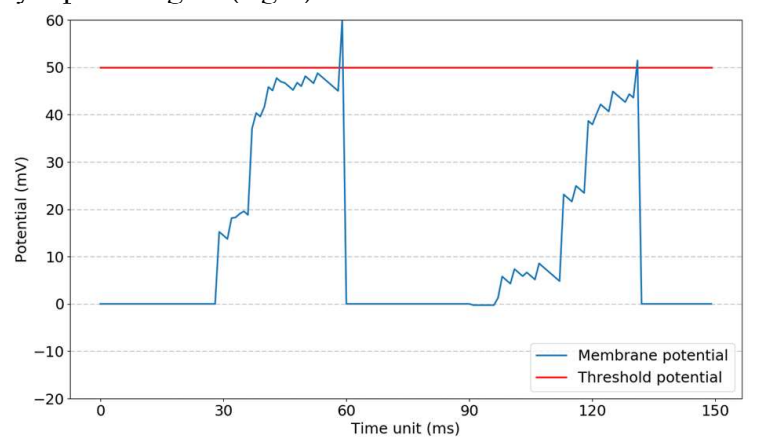

Figure 4 Corresponding voltage versus time figure of IF.

neurons increase or decrease its membrane potential. LIF model calculates the weighted sum of the voltage of each presynapse, and then compares it with the emission threshold. If the sum of the voltage exceeds the threshold, the current neuron fires a pulse. Otherwise, the neuron remains in resting state and does not emit pulse. The activated neurons will quickly enter into a refractory period in which no new input is allowed and the potential remains constant. In addition, a constant leakage of potential process is added when the potential of the neuron reaches a certain value. Figure 3 and figure 4 show a set of relationship between input pulses, synaptic weights and postsynaptic neuron voltage.

\subsection{Generation of spike trains}

In the biological vision system, single cell activity is modulated by spatial and temporal pattern of light to stimulate a certain region of the retina, and this region is called receptive field. For the input image of SNN, we design the sensing process of the receptive field by the convolution method. Manhattan distance is set as the convolution kernel in which the center of pattern increases membrane potential of the neural, as shown in matrix $\mathrm{M}$.

$$
M=\left[\begin{array}{ccccc}
-0.5 & -0.125 & 0.125 & -0.125 & -0.5 \\
-0.125 & 0.125 & 0.625 & 0.125 & -0.125 \\
0.125 & 0.625 & 1 & 0.625 & 0.125 \\
-0.125 & 0.125 & 0.625 & 0.125 & -0.125 \\
-0.5 & -0.125 & 0.125 & -0.125 & -0.5
\end{array}\right]
$$


Stimulus image, carrying the feature of low dimension is generated by convolution of input image. Stimulus calculated from its receptive field is an analog value and a corresponding conversion of information to artificial neuron spikes is needed. The spike generator serves as an encoder to convert this stimulus into spike train in the way of rate coding, so that neurons can understand it. It suggests that the information is carried by the firing rate of the neuron. The spike transmission process is simple and feasible. Firstly, set the same spike transmission period $\mathrm{T}$ for each pixel. Secondly, calculate corresponding spike transmission frequency according to the pixel value of stimulus image, and then generate a uniform spikes sequence according to it. That is, spike train generated has frequency proportional to the corresponding stimulus image.

\subsection{Study rule of Spike Time Dependent Plasticity (STDP)}

In 1949, Canadian psychologist Hebb found that it was possible to complete the learning of the neural system by changing the connection strength between neurons, which was called Hebbian learning theory. Hebbian theory refers to that the strength of connections between neurons can be modulated by the movements of neurons independently. In turn, changes in the degree of connection stimulate the posterior nerve, which echoes with each other and achieves the purpose of learning. The principle of STDP is a kind of Hebb learning mechanism, which is not symmetrical in time. It is caused by the temporal correlation of the action potentials between the presynaptic and postsynaptic neurons. When the action potential produced by presynaptic neurons is later than that produced by postsynaptic neurons, i.e. tpre >tpost, the correlation strength of synaptic connections will be weakened. On the contrary, when the action potential produced by presynaptic neurons is later than that produced by postsynaptic neurons, i.e. tpre <tpost, the strength of synaptic connections will be enhanced. The change of synaptic weight $\Delta \mathrm{w}$ is determined by the time difference tpre-tpost between the pre and post synaptic neurons. The expression of STDP function is as follows:

$$
\Delta \omega=\left\{\begin{array}{c}
A_{+} e^{\Delta t / \tau_{+}}, \Delta t<0 \\
-A_{-} e^{-\Delta t / \tau_{-}}, \Delta t>0
\end{array}\right.
$$

Among them, $\mathrm{A}_{+}$and $\mathrm{A}_{-}$represent the maximum enhancement and inhibition values of synaptic strength, both of which are positive. The time constant $\tau_{+}$and $\tau_{\text {- }}$ represents the time range of the STDP window for synaptic enhancement and inhibition.

In this process, the behavior of presynaptic neurons with high synaptic strength gives a stronger influence on the activity of postsynaptic neurons more than that with low synaptic strength. The behavior that presynaptic neuron attempts to control the action of postsynaptic neuron in order to initiate a fight between two neurons is called the competitiveness of STDP. This will enhance the synaptic strength between the strongly correlated postsynaptic and presynaptic neurons, while weakening the synaptic strength between the weakly correlated ones. The competitiveness of STDP leads to an infinite increase of synaptic strength, thus making the synapse itself unstable. In order to prevent the instability of synaptic strength, our method is to limit the range of synaptic weight to certain value $\omega_{\min }$ and $\omega_{\max }$.

\subsection{Lateral Inhibition}

Lateral inhibition is one of the unique information transmission rules of biological brain nerves. It disables the spreading of action potentials from excited neurons to neighboring neurons by lowers down the membrane potential of other neurons in same layer. This property is also called as Winner-Takes-All (WTA), which is held to enhance the perception of areas of contrast. In SNN, when a neuron reaches threshold voltage and generates an action potential, the potentials of other neurons in the same layer are forced to be set as suppression voltage.

\section{Experiment of SAR image classification based on SSN}

\subsection{MSTAR Dataset}

The experimental data used in this paper is the MSTAR data set collected by the SAR sensor of Sandia National Laboratory (SNL) [4]. In order to preliminarily explore the performance of SNN in SAR image classification, three categories of targets with the size of $128 \times 128$ pixels including armored vehicle BMP-2, BTR-60 and tank T-72, were selected and with certain limitation of aircraft heading angle. The category of targets, aircraft heading angle and corresponding quantity used in this experiment are shown in table I. The total number of samples is 236 , and the training process is completed by 50 cycles.

In order to completely measure the effectiveness of unsupervised learning algorithm based on SNN, the input images are SAR images without any preprocessing, and there is no ground truth information in the whole process of classification.

Table I Sample size of SAR target selected in the experiment

\begin{tabular}{|l|l|l|l|}
\hline $\begin{array}{l}\text { Category of } \\
\text { targets }\end{array}$ & BMP-2 & BTR-60 & T-72 \\
\hline $\begin{array}{l}\text { Minimum SAR } \\
\text { aircraft heading } \\
\text { angle }\end{array}$ & $13.191^{\circ}$ & $7.483^{\circ}$ & $-49.288^{\circ}$ \\
\hline $\begin{array}{l}\text { Maximum SAR } \\
\text { aircraft heading } \\
\text { angle }\end{array}$ & $73.191^{\circ}$ & $75.483^{\circ}$ & $10.712^{\circ}$ \\
\hline $\begin{array}{l}\text { Quantity of } \\
\text { samples }\end{array}$ & 92 & 52 & 92 \\
\hline
\end{tabular}
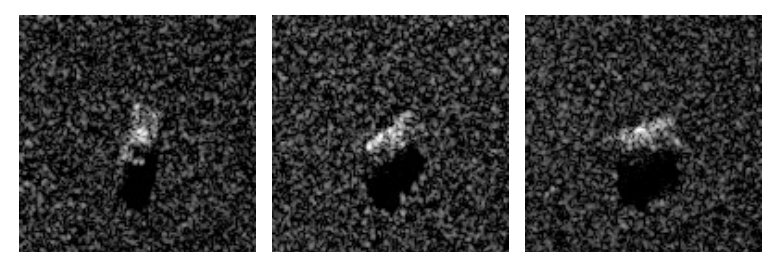

(a) 

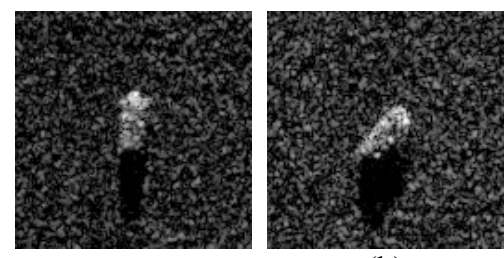

(b)
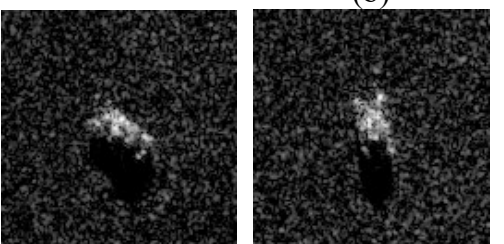

(c)
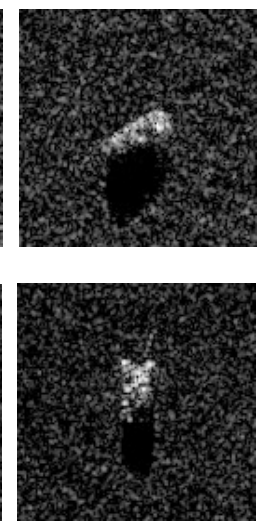

Figure 5 Three categories of SAR targets with certain limitation of aircraft heading angle. a) BMP-2, b) BTR-60, c) $\mathrm{T}-72$

\subsection{Accuracy and display of learning feature maps}

The unsupervised learning algorithm of SNN based on STDP divides the input SAR images into three categories. Each image represents a class and to each class a neuron is delegated. Spike counter gives out the winner by recording the total spikes in output neurons. Hence, we can determine the class to which the pattern belongs. In experiment, we consider that SNN has reached convergence state when the classification accuracy stagnates or fluctuates up and down. The learning process of SNN can be expressed by the curve of classification accuracy versus learning epoch, as shown in figure 6. Finally, the classification accuracy results for three categories of SAR targets are given in table II.

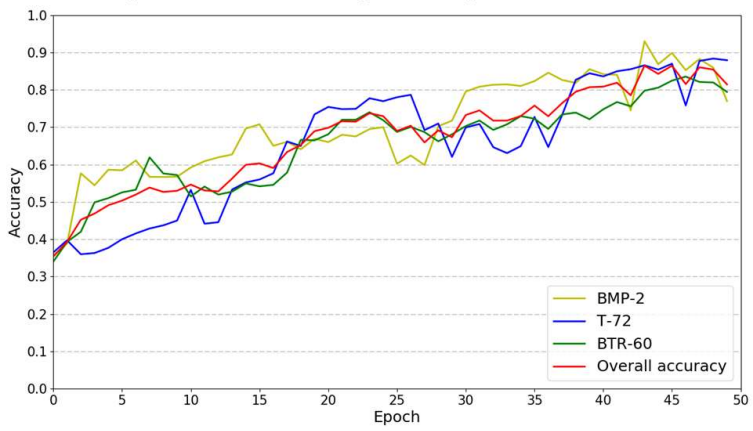

Figure 6 Classification accuracies of SAR target versus learning epoch based on SNN.

Table II Classification accuracy results of SAR target based on SNN

\begin{tabular}{|l|l|l|l|l|}
\hline Category & BMP-2 & BTR-60 & T-72 & $\begin{array}{l}\text { Overall } \\
\text { accuracy }\end{array}$ \\
\hline Accuracy & 0.853 & 0.819 & 0.854 & 0.842 \\
\hline
\end{tabular}

There is a unique generative property of SNN, which is used while demonstrating the results. After training SNN with MSTAR dataset, we scale to proper values and rearrange all the synapses connected to a particular output neuron in form of a $128 \times 128$ image. Figure 7 display learning feature maps of each output neuron, and help to depict what pattern that neuron has learned and how distinctly it can classify that pattern.
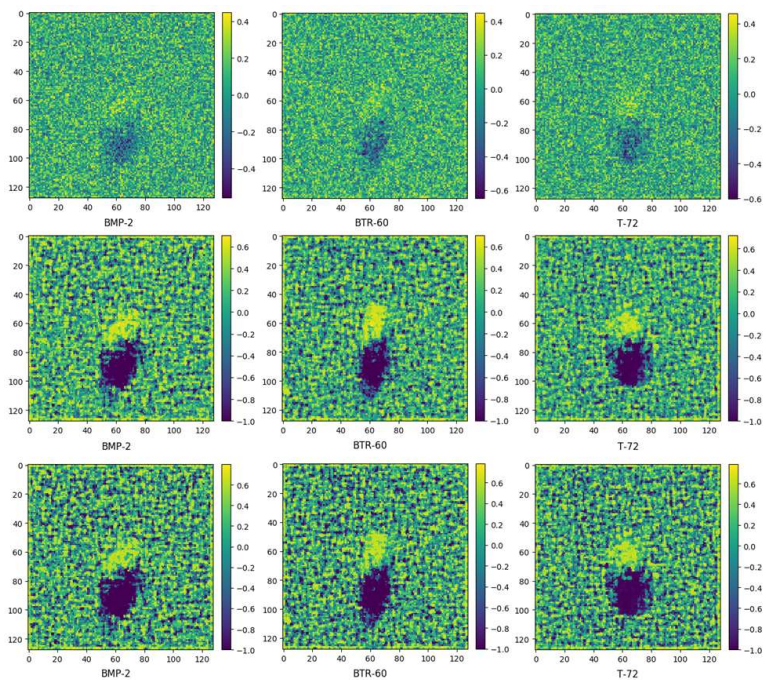

Figure 7 Visualization of learning features. (Epoch $=2$ for the first row, epoch=20 for the second row, epoch $=50$ for the third row)

\section{Conclusion}

In this paper, an unsupervised learning algorithm of STDP based on the spiking neural network is proposed. The LIF neural model is adopted and all parameters of the neural structure are within the range of biological rationality. Meanwhile, the learning and updating process of synaptic weight is completed by combining with the rule of STDP, so as to better retain the competitiveness and stability of synapses. It has been proved that the synaptic weights in SNN could be dynamically updated by STDP rule without any supervision. Finally, a verification experiment of SNN was carried out on MSTAR data set and the overall classification accuracy of SAR targets reached $84.2 \%$, which showed the potential and efficiency of the architecture. The general property of SNN further verifies its learning adaptability and feature expression ability to SAR images.

\section{$6 \quad$ Literature}

[1] Morrison A, Diesmann M, Gerstner W. Phenomenological models of synaptic plasticity based on spike timing[J]. Biological cybernetics, 2008, 98(6): 459478.

[2] Diehl P U, Neil D, Binas J, et al. Fast-classifying, high-accuracy spiking deep networks through weight and threshold balancing[C]//2015 International Joint Conference on Neural Networks (IJCNN). IEEE, 2015: 1-8.

[3] Zhang T, Zeng Y, Zhao D, et al. A plasticity-centric approach to train the non-differential spiking neural networks[C]//Thirty-Second AAAI Conference on Artificial Intelligence. 2018.

[4] Keydel E R. MSTAR extended operating conditions: A tutorial[C]. Algorithms for Synthetic Aperture Radar Imagery III, Orlando, F. L., USA, 1996: 228 242. 